\title{
Transient Myocardial Ischemia of Newborn
}

National Cancer Institute

\section{Source}

National Cancer Institute. Transient Myocardial Ischemia of Newborn. NCI Thesaurus. Code C112865.

A condition characterized by a temporary imbalance between the oxygen supply and demand of the heart muscle in the newborn. 